\title{
PRPF6 Induces the Alternative Splicing of IncRNA SNHG16 To Promote Progression and Paclitaxel Resistance of Ovarian Cancer via Regulating GATA3 Transcription
}

\section{Han Wang}

Shengjing Hospital of China Medical University

Yingying Zhou

Shengjing Hospital of China Medical University

Siyang Zhang

Shengjing Hospital of China Medical University

Ya Qi

Shengjing Hospital of China Medical University

Min Wang ( $\nabla$ wm21st@126.com )

Shengjing Hospital of China Medical University https://orcid.org/0000-0002-8963-5808

\section{Research}

Keywords: PRPF6, SNHG16, paclitaxel, ovarian cancer

Posted Date: November 15th, 2021

DOI: https://doi.org/10.21203/rs.3.rs-1045802/v1

License: (c) (i) This work is licensed under a Creative Commons Attribution 4.0 International License.

Read Full License 


\section{Abstract \\ Background}

Small nucleolar RNA host gene 16 (SNHG16) and pre-mRNA processing factor 6(PRPF6) play vital roles in regulatory mechanisms of multiple cancers, but the mechanisms in ovarian cancer (OC) remains poorly understood.

\section{Methods}

The expression of SNHG16 transcripts-SNHG16-L/S in OC tissues were analyzed by real-time PCR (RTPCR). The expression of PRPF6 in OC tissues were detected by Immunohistochemistry (IHC).

Tumorigenesis, epithelial-to-mesenchymal transition (EMT) and PTX-resistance were detected by western blot, transwell, CCK-8 assays, colony formation assays and flow cytometry analyses. Molecular interactions were examined by dual-luciferase reporter gene assay, RNA immunoprecipitation (RIP) and chromatin immunoprecipitation (ChIP).

\section{Results}

The results indicated the expression of SNHG16-L/S was opposite in chemo-resistance and chemosensitivity tissues of OC. And SNHG16-L/S had different effects on the progression and PTX-resistance of OC cells. SNHG16-L inhibited GATA binding protein 3 (GATA3) transcription through CCAAT/enhancerbinding protein $b$ (CEBPB) to further promote tumorigenesis, EMT and PTX-resistance of OC. Moreover, PRPF6 was upregulated in chemo-resistance tissues of OC. PRPF6 promoted tumorigenesis and PTXresistance in vitro and in vivo. Mechanistically, PRPF6 induced the alternative splicing of SNHG16 to downregulate SNHG16-L, which further mediated progression and PTX-resistance through upregulating GATA3 in OC.

\section{Conclusions}

Totally, the results demonstrated that PRPF6 promoted progression and PTX-resistance in OC through SNHG16-L/CEBPB/GATA3 axis. Thus, PRPF6 may become a valuable target for OC therapy.

\section{Background}

According to the global cancer statistics from the World Health Organization, 313,959 new cases of ovarian cancer (OC) and 207,252 OC-related deaths were reported in 2020 worldwide[1, 2]. Treatment of advanced OC is surgery with platinum and Paclitaxel (PTX) chemotherapy[3]. The potential pathological mechanisms of tumorigenesis and PTX-resistance in OC may be very complicated, and the current research has not reached a consensus. Despite the advances in $\mathrm{OC}$ diagnosis and treatments, the poor 
prognosis and high recurrence rate are still the main obstacles $[4,5]$. Therefore, it is essential to explore key factors contributing to tumorigenesis and PTX-resistance of OC, and to seek novel strategies for OC patients.

Long noncoding RNA (IncRNA) has implicated in the pathogenesis of many cancers[6-10], and can participate in transcriptional regulation and epigenetic modification[11-13]. SNHG16 (small nucleolar RNA host gene 16) as a IncRNA, has been proved to be involved in mechanisms of various cancers[1416]. SNHG16 regulates miR-216A-5p/ZEB1 axis to induce epithelial-to-mesenchymal transition (EMT) in cervical cancer[14]. However, the molecular mechanism of SNHG16 in OC still at an early stage.

RNA binding proteins (RBPs) can bind to double or single strand RNA to further control RNA metabolism. RBPs are involved in RNA splicing, transcription and translation. Alternative splicing (AS) is dependent on RBPs, which refers to pre-mRNA was spliced to produce different mRNA splicing isomers by different splicing methods. Aberrant AS involves in the development of multiple cancers[17]. Notably, recent studies demonstrate that different isoforms of IncRNAs exhibit distinct, even opposite function in tumorigenesis. MBNL3 regulates AS of IncRNA PXN-AS1 and further promote the inclusion of exon 4 to upregulate PXN in hepatocellular cancer[18]. This provides novel direction for future research on RBPs and IncRNAs.

Pre-mRNA processing factor 6(PRPF6), as an RBP, participates in the regulation of AS, increases the expression of isoforms with oncogene function, and promotes tumor proliferation and metastasis[1926]. PPRF6 regulates AS of an oncogenic form of the ZAK kinase to drive proliferation in colon cancer[21]. PRPF6 activates oncogenic AR/AR-Vs to promote progression of hepatocellular cancer and prostate cancer[23, 24]. However, no concerned study has been reported about PRPF6 in OC.

EMT features decrease in E-cadherin, while an increase in N-cadherin and vimentin. Recent research proves that the invasion of cancer cells resulted from EMT-mediated metastasis can lead to PTX resistance. The upstream mediators of EMT, such as ZEB1/2, microRNAs, TGF- $\beta$, are involved in regulating PTX-resistance[27, 28]. Moreover, the mutation of $\beta$-tubulin III and interfering with the polymerization of microtubules is the prominent mechanism of PTX-resistance in OC[29].

In our study, IncRNA arrays suggested that SNHG16 isoforms were differentially expressed in OC cell line SKOV3 and PTX-resistant OC cell line SKOV3-TR30, which is defined as SNHG16-L (SNHG16-001, ENST00000448136.5) and SNHG 16-S (SNHG16-002, ENST00000590435.5). We revealed PRPF6 promoted progression and PTX-resistance in OC. Furthermore, PRPF6 induced AS of SNHG16 to downregulate SNHG16 expression, which further regulated transcription activity of GATA binding protein 3 (GATA3) and potentiated EMT pathway in OC. Thus, PRPF6 may be a valuable target for OC therapy.

\section{Methods}

\section{Patients and tissues}


Ovarian serous cancer tissue samples were collected from a total of 40 patients from 2017-2020 in Department of Gynecology and Obstetrics of Shengjing Hospital of China Medical University. At least two pathology experts jointly determined the postoperative pathology of in all the cases. Of these, 30 chemoresistance cases and 102 chemo-sensitivity cases were determined according to NCCN guidelines. All patients have provided signed the informed consent and the experimental protocol was approved by the Institutional Medical Research Ethics Committee of the Shengjing Hospital of China Medical University(2020PS274K-X1).

\section{Cell culture}

SKOV3 and 293T was obtained from the were acquired from the Tumor Cell Bank of the Chinese Academy of Medical Sciences (Beijing). The PTX-resistant OC cell line, SKOV3-TR30 was derived from SKOV3 and provided by Zhejiang University affiliated Obstetrics and Gynecology Hospital (Hangzhou). SKOV3 and SKOV3-TR30 cell lines were cultured in RPMI 1640 (Hyclone, USA). SKOV3-TR30 cells were maintained with the addition of $20 \mathrm{nM}$ of PTX (Sigma Aldrich, MO). 293T cells were cultured in DMEM (Hyclone, USA). All the medium contains $10 \%$ fetal bovine serum (FBS, Procell) and $1 \%$ penicillin/streptomycin. Cells were cultured at $37^{\circ} \mathrm{C}$ with $5 \% \mathrm{CO} 2$.

\section{Real-time PCR(RT-PCR)}

Total RNA was isolated using Trizol reagent (Invitrogen). cDNA was synthesized according to the manufacturer's protocol (Takara). SYBR premix Ex TaqTM II (Takara) was used for PCR. The primers were synthesized by Sangong (Shanghai, China) and shown as follows: PRPF6: forward:

GAGGATGCTGACAGTTGTGTAG, reverse: CCATGGTTCTTCTCGAAGTACG; SNHG16-L: forward: CCAGTTACACAGGATGCCGTCTTG, reverse: AGCTGATTGCCTTGGTGAGTCAAC; SNHG16-S: forward: GCCAAGGTGAAGCGAGCTGAG, reverse: GCAAGAGACTTCCTGAGGCACAT; CEBPB: forward: GCACAGCGACGAGTACAAGA, reverse: TGCTTGAACAAGTTCCGCAG; GATA3: forward: GTCCTGTGCGAACTGTCAGA, reverse: CGAGCTGTTCTTGGGGAAGT. The relative expression of RNAs was normalized by $2-\triangle \triangle C T$ method.

\section{Cell transfection}

si-RNAs were synthesized from Ribobio (Guangzhou, China). The sequences were as follows: si-PRPF6001: GAAGCGGGTTCTTCGGAAA, si-PRPF6-002: GGATCTAAATGACACCAAT, si-PRPF6-003: CTCGGAACCTTATCATGAA. The overexpression plasmids pHBLV-PRPF6, pcDNA3.1-SNHG16-L, pcDNA3.1-SNHG16-S, pcDNA3.1-CEBPB, and pHBLV-h-GATA3 were synthesized from Hanbio (Shanghai, China). Lipofectamine 3000 (Invitrogen) was used transfection according to the instructions.

\section{Transwell assay}

$5 \star 104$ cells were suspended in a serum-free medium and plated on upper transwell migration chambers (Corning Costar). Transwell invasion assay was coated with Matrigel (BD). The lower chambers added medium with $10 \%$ FBS. After cultured 48 h, the membranes were fixed with methanol and stained with $1 \%$ 
crystal violet. Five random fields ( $\times 400$ magnifications) were counted and photographed under the light microscope.

\section{Cell Counting Kit-8 (CCK8) assay and cell viability assay}

50000 cells were seeded in 96 -well plate with 100 ul medium per well. We added 10 ul CCK8 reagent (Sigma) at $0,24,48,72$ and $96 \mathrm{~h}$. The optical density was measured at $450 \mathrm{~nm}$. For cell viability assays, we added various concentrations of PTX after seeded for $24 \mathrm{~h}$. Then, added $10 \mathrm{ul} \mathrm{CCk8} \mathrm{reagent} \mathrm{at} 48 \mathrm{~h}$ and examined in 2 hours.

\section{Colony Formation Assay}

Transfected OC cells were plated in 6-well plates and incubated for 10 days (1000 cells/well). Then the cells in the well were fixed with methanol and stained with $0.1 \%$ of crystal violet. Then calculated by counting the number of visible colonies.

\section{Cell apoptosis assay}

Cells were digested with EDTA-free trypsin. The cells were stained with Annexin V-FITC/PI or Annexin VPE/7-AAD (for cells transfected with green fluorescence plasmid) (BestBio). Flow cytometry was used to analysis apoptosis.

\section{Western blot}

Total protein was extracted via RIPA lysis (Beyotime) with phenyl-methane-sulfonyl fluoride and protease inhibitor. $10 \%$ SDS-PAGE gel electrophoresis with $30 \mu \mathrm{g}$ protein per well was performed and then we transferred PVDF membranes. Antibodies PRPF6 (1:2000, Abcam), CEBPB (1:2000, Abcam), GATA3(1:2000, Abcam), Vimentin (1:1000, Elabscience), E-cadherin (1:1000, Elabscience), N-cadherin (1:1000, Elabscience), $\beta$-tubulin III (1:2000, Immunoway), $\beta$-actin (1:5000, Bioworld) were incubated overnight at $4^{\circ} \mathrm{C}$.

\section{RNA immunoprecipitation (RIP)}

The experiment was conducted followed the manufacturer's protocol of Magna RIP kit (Millipore). The antibodies used for RIP were PRPF6(10ug per reaction, Abcam) and CEBPB (10ug per reaction, Abcam). RNA was extracted after detachment from the bead using protease $\mathrm{K}$. The expression of SNHG16 premRNA, SNHG16-L and SNHG16-S was determined using RT-qPCR.

\section{Chromatin immunoprecipitation (ChIP)}

ChIP was performed according to the instructions of EZ ChIP KIT(Millipore). The antibodies used for ChIP were CEBPB (10ug, Abcam). The possible binding sites of CEBPB and GATA3 promoter were predicted via Jaspar, and specific primers were synthesized by Sangong (Shanghai, China), sequences were as follows: GATA3 promoter: forward: CAAGCCCTTTGCCCCAT, reverse: CAGGTAGAGTTTTCCCTTCACAA. The enrichment of GATA3 promoter was detected by RT-PCR.

\section{Dual-luciferase reporter gene assay}


The luciferase plasmids pSI-Check2-GATA3 wild type/mutant type(wt-GATA3/mut-GATA3) were synthesized by Hanbio (Shanghai, China). According to the instruction of Dual-Luciferase® Reporter Assay System (Promega), the luciferase activity was detected.

\section{Immunohistochemistry (IHC)}

The paraffin sections were deparaffinized and antigen retrieval was performed by adding citrate buffer ( $\mathrm{pH}$ 6.1). The sections were then incubated with PRPF6 antibodies (Abcam, 1:250) were diluted in 5\% BSA, followed by DAB staining (Elabscience) and observation under microscope.

\section{Immunofluorescence (IF)}

Cells growing on coverslips in 6-well plates were removed and fixed with $4 \%$ paraformaldehyde. Then, permeabilized in $0.5-1.0 \%$ Triton X-100 for 10 min and blocked with 5\% BSA for 30 minutes. The cells were then incubated with antibodies PRPF6 (1:150, Abcam), CEBPB (1:150, Abcam), GATA3(1:150, Abcam) overnight at $4^{\circ} \mathrm{C}$. Fluorescent-labelled secondary antibody (1:100, Proteintech) was added and incubated in the dark for $2 \mathrm{~h}$. Cells were stained with DAPI and observed under the fluorescent microscope.

\section{Fluorescence in situ Hybridization (FISH)}

Cells growing on coverslips in 6-well plates were removed. The SNHG16 probe were synthesized by Servicebio. The experiment was operated according to the protocol of Fluorescent in Situ Hybridization Kit (Ribobio). The slips observed under the fluorescence microscope.

\section{Xenografts in nude mice}

The lentivirus containing siPRPF6 sequence was synthesized by Gene-Pharma (Shanghai, China). The SKOV3-TR30 cells were infected with lentivirus and obtained stably transfected cells. 4-weeks-old female BALB/cA-nu Mice(N=3/group) were purchased from Huafukang (Beijing, China). The mice subcutaneously inoculated with stable transfected cell suspension (200 $\mu \mathrm{L}, 5 \times 10^{6}$ cells) into dorsal part to observe tumor growth. After 1 week, PTX $(20 \mathrm{mg} / \mathrm{kg})$ or saline was injected into tumor every 3 days for 3 weeks when tumor size reached $80-100 \mathrm{~mm}^{3}$. Animal experiments were performed according to the ethical guidelines for animal experiments and were approved by China Medical University Animal Welfare and Ethical Community (CMU2020341).

\section{Statistical analysis}

The statistics were analyzed with SPSS 22.0. The results represented as the mean \pm standard deviation (SD). Data with normal distribution and homogeneity of variance were compared by paired sample t-test or non-paired t-test. One-way analysis of variance (ANOVA) was used for comparison among multiple groups. Repeated measures ANOVA, followed by the Bonferroni post hoc test, were used to analyze multiple groups at different time points. Significantly difference was set as $\mathrm{P}<0.05$.

\section{Results}




\section{SNHG16-L/S were expressed opposite in OC and closely related to FIGO stage and differentiation}

Previous IncRNA arrays suggested that the transcripts of SNHG16 were differentially expressed in chemosensitive OC cell line SKOV3 and chemo-resistant OC cell line SKOV3-TR30(Figure.1A). ENSEMBL's annotation showed the difference of SNHG16-L/S is the existence of exon 1 (Figure.1B). The expression of SNHG16-L in SKOV3-TR30 was lower than that in SKOV3, and the expression of SNHG16-S was opposite (Figure.1C). SNHG16-L/S was differential expressed in OC tissues as detected by RT-PCR, which showed SNHG16-S was relatively high in chemo-resistant groups, while SNHG16-L was the opposite (Figure.1D). Then, we analyzed the relationship between SNHG16-L/S expression and clinical characteristics. SNHG16-L/S expression were closely related to FIGO stage and differentiation, and were not related to age and lymph node metastasis (Table 1).

\section{SNHG16-L/S had different effect on the tumorigenesis, EMT and PTX-resistance of OC}

To further examine the roles of SNHG16-L/S in the development and chemoresistance in OC, we stably transfected OE-SNHG16-L in SKOV3-TR30 and OE-SNHG16-S in SKOV3 (Supplementary Fig. 1). Transwell assays confirmed that the presence of OE-SNHG16-L resulted in a decrease in cell migration and invasion in SKOV3-TR30, whereas OE-SNHG16-S resulted in an increase in SKOV3 (Figure.2A). The OE-SNHG16-L in SKOV3-TR30 and OE-SNHG16-S in SKOV3 cells, decreased and increased cell proliferation, respectively, as judged by CCK-8 assays and colony formation assays (Figure.2B\&C). In addition, the flow cytometry analysis showed that OE-SNHG16-L promoted cell apoptosis, whereas OE-SNHG16-S inhibited cell apoptosis (Figure.2D). OE-SNHG16-L inhibited PTX-resistance, while OE-SNHG16-S promoted PTXresistance examined by various doses of PTX via cell viability assays (Figure.2E). Western blot analysis showed that OE-SNHG16-L decreased the expression of N-cadherin, $\beta$-tubulin III and vimentin, and increased E-cadherin expression, while OE-SNHG16-S prompted the opposite result (Figure.2F). All of these data suggested that SNHG16-L inhibited tumorigenesis and PTX-resistance in SOC, while SNHG16S appeared opposite results.

\section{SNHG16-L downregulated GATA3 expression to inhibit EMT and PTX-resistance of OC}

The LncMAP database (http://bio-bigdata.hrbmu.edu.cn/LncMAP) suggested that SNHG16 may regulate GATA3 via transcriptional factor CEBPB in OC (Figure.3A). SNHG16 was located in the nucleus detected by FISH(Figure.3B). OE-GATA3 in SKOV3 cells resulted in upregulated in $\mathrm{N}$-cadherin, vimentin, and $\beta$ tubulin III and downregulated E-cadherin, detected by Western blot (Figure.3C). Transfection of OESNHG16-L in SKOV3-TR30 cells inhibited GATA3, N-cadherin, vimentin and $\beta$-tubulin III expression and promoted E-cadherin. The effect was reversed after transfected OE-SNHG16-L+OE-GATA3, confirmed by western blot (Figure.3D). These results demonstrated that SNHG16-L downregulated GATA3 expression to inhibit EMT and PTX- 


\section{SNHG16-L combined with CEBPB and inhibited GATA3's transcriptional activity in OC}

CatRAPID omics (http://service.tartaglialab.com/page/catrapid_omics_group)indicated that SNHG16-L combined with CEBPB protein (Figure.4A). RIP test using the CEBPB antibody was conducted in SKOV3TR30 cells. The result showed that CEBPB protein complex was precipitated. Through synthesizing different primers, it was found that CEBPB could bind to SNHG16-L, while SNHG16-S and CEBPB did not have this binding effect (Figure.4B). Immunofluorescence detection showed that the expression of CEBPB and GATA3 were located in the nucleus (Figure.4C). The sequence analysis showed a potential binding site for CEBPB in the promoter region of GATA3 gene (Figure.4D). ChIP detection in SKOV3-TR30 using antibody of CEBPB were carried out. The results showed that compared with IgG-bound samples, CEBPBbound complexes significantly enriched the 898-907bp region of GATA3 promoter (Figure.4E). Then, dualluciferase reporter gene assay showed that CEBPB overexpression enhanced luciferase activity of GATA3wt group but not GATA3-mut group (Figure.4F). The enhancement was weakened after overexpression of SNHG16-L(Figure.4G). These data identified that SNHG16-L combined with CEBPB and inhibited GATA3's transcriptional activity in OC.

PRPF6 was upregulated in chemo-resistant tissues and was related to FIGO stage, differentiation and lymph node metastasis of OC

To further analysis whether SNHG16-L/S were mediated by AS, catRAPID omics analysis found that 413$419 \mathrm{bp}$ of SNHG16 pre-mRNA exon 1 may be a binding site with PRPF6 (Figure.5A). PRPF6 was relative upregulated in SKOV3-TR30 compared with SKOV3, as detected by RT-PCR (Figure.5B). IHC was used to detect PRPF6 expression in OC tissues. PRPF6 was relatively higher in chemo-resistance OC tissues $(\mathrm{N}=30)$ than that in chemo-sensitivity tissues $(\mathrm{N}=102$, Figure.5C). Then, we investigated relationship between PRPF6 expression and clinicopathological characteristics. The results showed that PRPF6 was closely related to FIGO stage, differentiation and lymph node metastasis (Table 2).

\section{PRPF6 promoted tumorigenesis, EMT and PTX-resistance in $\mathrm{OC}$ cells and in vivo}

Three si-PRPF6 was transfected in SKOV3-TR30 cells and pc-DNA3.1-PRPF6 was stably transfected in SKOV3 cells. si-PRPF6-003 had the highest silencing efficiency and was named as si-PRPF6 (Supplementary Fig. 1). To study the function of PRPF6, CCK assays showed that si-PRPF6 and OEPRPF6 significantly inhibited and promoted cell growth, respectively (Figure.6A). Colony Formation Assay also showed similar effect (Figure.6B). Transwell assays indicated si-PRPF6 resulted in a decrease in invasion and migration, while OE-PRPF6 resulted in an increase (Figure.6C). The cell viability assays treated with various doses of PTX indicated si-PRPF6 inhibited PTX resistance, while OE-PRPF6 promoted PTX resistance (Figure.6D). The flow cytometry showed si-PRPF6 induced apoptosis, and OE-PRPF6 was the opposite (Figure.6E). Then, we further explored the effects of PRPF6 in vivo. The stable cell lines constructed by lentivirus containing siPRPF6 sequence in SKOV3-TR30 were injected in 4-weeks-old 
female BALB/cA-nu Mice(N=3/group). These mice were divided into 4 groups (Figure.6F). After a week, PTX (20 mg/kg) or saline was injected into tumor every 3 days for 3 weeks. Compared with NC, the results showed that sh-PRPF6 inhibited tumor growth and weight (Figure.6G\&H). si-PRPF6 in SKOV3TR30 cells inhibited GATA3, N-cadherin, vimentin and $\beta$-tubulin III expression and upregulated E-cadherin detected by western blot (Figure.6I). Taken together, the data revealed that PRPF6 promoted migration, invasion, EMT and PTX-resistance in OC cells and in vivo.

\section{PRPF6 induced AS of SNHG16 to upregulate GATA3 expression.}

Depletion of PRPF6 upregulated SNHG16-L and downregulated SNHG16-S, while overexpression of PRPF6 had the opposite effect. These results suggested that PRPF6 could induce AS of SNHG16 (Figure.7A). PRPF6 was located in the nucleus examined by Immunofluorescence detection (Figure.7B). RIP test using the PRPF6-specific antibody was conducted in over expressed PRPF6 SKOV3-TR30 cells. By synthesizing SNHG16 pre-mRNA and SNHG16-L/S primers, the results showed that PRPF6 can combine with SNHG16-L and SNHG16 pre-mRNA, not with SNHG16-S (Figure.7C). IHC was used to detect GATA3 expression in shNC and shPRPF6 groups of mice tissues (Figure.7D). OE-PRPF6 increased GATA3, $\mathrm{N}$-cadherin, $\beta$-tubulin III and vimentin and decreased E-cadherin expression level. While OE-SNHG16-L appeared the opposite (Figure.7E). Taken together, these data implicated that PRPF6 promoted AS of SNHG16, upregulated SNHG16-S and downregulated SNHG16-L. SNHG16-L further inhibited transcription activity of GATA3 via binding to CEBPB to promote EMT and PTX-resistance in OC(Figure.7F).

\section{Discussion}

In this study, we found that SNHG16 transcripts had opposite expression in OC. The pattern of SNHG16 has been could be upregulation and downregulation in multiple cancers[30-35]. SNHG16 can activating WNT/ $\beta$-catenin pathway and EMT in cervical cancers[35]. Interestingly, our findings seem to be first illustrated the different effects of SNHG16-L/S in OC. We then evaluated biological functions of SNHG16L/S in OC cells. Through several function assays, we further identified SNHG16-L overexpression inhibited cell progression, metastasis, EMT and PTX-resistance in OC, while SNHG16-S was the opposite. LncRNA can mediate transcription regulation by affecting binding of transcription factors and target genes. SNHG16 can recruit SPI1 protein to promote its transcriptional activation of PARP9 promoter in cervical cancer[36]. Interestingly, our study found that CEBPB directly bound to GATA3 promoter. SNHG16$L$ bound to CEBPB to further regulate GATA3 transcription.

CCAAT/enhancer-binding protein $b$ (CEBPB) plays a vital role in cell proliferation and differentiation, metabolism and tumor development[37, 38]. CEBPB, as a DNA binding protein, can enhance the activity of H3K79 methyltransferase DOT1L and regulate the methylation of $\mathrm{H} 3 \mathrm{~K} 79$ to promote the occurrence of cisplatin resistance in OC[39]. Moreover, CEBPB can block the transcription regulation of GDF15 and this effect can be inhibited by IncRNA GAS5 in OC[40]. GATA3 has proved highly expressed in multiple OC cell lines, which is related to poor prognosis and induced EMT[41-45]. The high expression of GATA3 increases the ratio of $p$-p38MAPK/p-ERK, and promotes the stemness and Paclitaxel (PTX) resistance of 
OC[44]. In this study, we reported that GATA3 facilitated EMT and PTX-resistance in OC, which is consistent with previous literature findings.

To further investigate whether the differential effect of SNHG16 was induced by AS. We found PRPF6 may be a potential splicing factor related to SNHG16. PRPF6 acts as an oncogene in colorectal cancer, prostate cancer and hepatocellular cancer[23, 24]. The function of PRPF6 has not been reported in the other cancers, including OC. Our study first reported PRPF6 was upregulated in chemoresistance tissues of OC, and was closely related to FIGO stage, differentiation and lymph node metastasis. PRPF6 promoted progression, metastasis, EMT and PTX-resistance of OC cells in vitro and in vivo.

Furthermore, our study suggested that PRPF6 binds to SNHG16 pre-mRNA and SNHG16-L instead of SNHG16-S, which indicated that PRPF6 is possibly recruited to exon 1. AS has 7 basic patterns, such as exon skip, retained intron, alternate donor site, etc. RNA binding proteins bind to adjacent splice sites and promote the recruitment of other spliceosomes[46]. These data indicates that the splicing pattern of SNHG16 may be exon 1 skip. However, the specific mechanism and sites of AS remains to be investigated in-depth in future studies.

\section{Conclusions}

Collectively, our study demonstrated that PRPF6 promoted progression, EMT and PTX-resistance via SNHG16-L/CEBPB/GATA3 axis in OC. Our findings show the significant role of PRPF6, which may as novel biomarkers for OC therapy.

\section{Abbreviations}

$\begin{array}{ll}\text { PTX } & \text { Paclitaxel } \\ \text { PRPF6 } & \text { Pre-mRNA processing factor } 6 \\ \text { SNHG16 } & \text { small nucleolar RNA host gene 16 } \\ \text { RIP } & \text { RNA immunoprecipitation } \\ \text { ChIP } & \text { chromatin immunoprecipitation } \\ \text { CEBPB } & \text { CCAAT/enhancer-binding protein } \beta \\ \text { GATA3 } & \text { GATA binding protein } 3 \\ \text { EMT } & \text { epithelial-to-mesenchymal transition } \\ \text { RT-PCR } & \text { Real-time PCR } \\ \text { CCK8 } & \text { Cell Counting Kit-8 }\end{array}$

\section{Declarations}




\section{Ethics approval and consent to participate}

All patients have provided signed the informed consent and the experimental protocol was approved by the Institutional Medical Research Ethics Committee of the Shengjing Hospital of China Medical University(2020PS274K-X1). Animal experiments were performed according to the ethical guidelines for animal experiments and were approved by China Medical University Animal Welfare and Ethical Community (CMU2020341).

\section{Consent for publication}

Not applicable.

\section{Availability of data and materials}

The datasets used and analyzed during the current study are available from the corresponding author on reasonable request.

\section{Competing interests}

The authors declare that they have no competing interests.

\section{Funding}

This work is supported by The Outstanding Scientific Fund of Shengjing Hospital (Grant No.201705).

\section{Authors' contributions}

Han Wang, Yingying Zhou and Min Wang designed the experiments. Han Wang analyzed and interpreted the data of the experiments. Han Wang, Siyang Zhang and Ya Qi performed the experiments. The first draft was written by Han Wang. All authors read and approved the final manuscript.

\section{Acknowledgements}

Not applicable

\section{References}

1. Sung H, Ferlay J, Siegel RL, Laversanne M, Soerjomataram I, Jemal A, Bray F: Global Cancer Statistics 2020: GLOBOCAN Estimates of Incidence and Mortality Worldwide for 36 Cancers in 185 Countries. CA: a cancer journal for clinicians 2021, 71(3):209-249.

2. Siegel RL, Miller KD, Jemal A: Cancer statistics, 2020. CA: a cancer journal for clinicians 2020, 70(1):7-30.

3. Vaughan S, Coward JI, Bast RC, Jr., Berchuck A, Berek JS, Brenton JD, Coukos G, Crum CC, Drapkin R, Etemadmoghadam $\mathrm{D}$ et al: Rethinking ovarian cancer: recommendations for improving outcomes. 
Nature reviews Cancer 2011, 11(10):719-725.

4. Orr GA, Verdier-Pinard P, McDaid H, Horwitz SB: Mechanisms of Taxol resistance related to microtubules. Oncogene 2003, 22(47):7280-7295.

5. Monk BJ, Herzog TJ, Tewari KS: Evolution of Chemosensitivity and Resistance Assays as Predictors of Clinical Outcomes in Epithelial Ovarian Cancer Patients. Current pharmaceutical design 2016, 22(30):4717-4728.

6. Guttman M, Rinn JL: Modular regulatory principles of large non-coding RNAs. Nature 2012, 482(7385):339-346.

7. Adams BD, Anastasiadou E, Esteller M, He L, Slack FJ: The Inescapable Influence of Noncoding RNAs in Cancer. Cancer Res 2015, 75(24):5206-5210.

8. Shen $Y$, Peng $X$, Shen $C$ : Identification and validation of immune-related IncRNA prognostic signature for breast cancer. Genomics 2020, 112(3):2640-2646.

9. Kong X, Duan Y, Sang Y, Li Y, Zhang H, Liang Y, Liu Y, Zhang N, Yang Q: LncRNA-CDC6 promotes breast cancer progression and function as ceRNA to target CDC6 by sponging microRNA-215. Journal of cellular physiology 2019, 234(6):9105-9117.

10. Luo H, Xu C, Le W, Ge B, Wang T: IncRNA CASC11 promotes cancer cell proliferation in bladder cancer through miRNA-150. J Cell Biochem 2019, 120(8):13487-13493.

11. Ye M, Wang C, Zhu J, Chen M, Wang S, Li M, Lu Y, Xiao P, Zhou M, Li X et al: An NF-kB-responsive long noncoding RNA, PINT, regulates TNF-a gene transcription by scaffolding p65 and EZH2. FASEB journal: official publication of the Federation of American Societies for Experimental Biology 2021, 35(9):e21667.

12. Yin X, Yang J, Chen J, Ni R, Zhou Y, Song H, Jin L, Tang T, Pan Y: LncRNA CTD-3252C9.4 modulates pancreatic cancer cell survival and apoptosis through regulating IFI6 transcription. Cancer cell international 2021, 21(1):433.

13. Li W, Li Y, Zhang H, Liu M, Gong H, Yuan Y, Shi R, Zhang Z, Liu C, Chen C et al: HOTAIR promotes gefitinib resistance through modification of EZH2 and silencing p16 and p21 in non-small cell lung cancer. Journal of Cancer 2021, 12(18):5562-5572.

14. Zhu H, Zeng Y, Zhou CC, Ye W: SNHG16/miR-216-5p/ZEB1 signal pathway contributes to the tumorigenesis of cervical cancer cells. Archives of biochemistry and biophysics 2018, 637:1-8.

15. Liu Y, Gu S, Li H, Wang J, Wei C, Liu Q: SNHG16 promotes osteosarcoma progression and enhances cisplatin resistance by sponging miR-16 to upregulate ATG4B expression. Biochemical and biophysical research communications 2019, 518(1):127-133.

16. Xu P, Xiao H, Yang Q, Hu R, Jiang L, Bi R, Jiang X, Wang L, Mei J, Ding F et al: The USP21/YY1/SNHG16 axis contributes to tumor proliferation, migration, and invasion of non-smallcell lung cancer. Experimental \& molecular medicine 2020, 52(1):41-55.

17. Urbanski LM, Leclair N, Anczuków O: Alternative-splicing defects in cancer: Splicing regulators and their downstream targets, guiding the way to novel cancer therapeutics. Wiley Interdiscip Rev RNA 2018, 9(4):e1476. 
18. Yuan JH, Liu XN, Wang TT, Pan W, Tao QF, Zhou WP, Wang F, Sun SH: The MBNL3 splicing factor promotes hepatocellular carcinoma by increasing PXN expression through the alternative splicing of IncRNA-PXN-AS1. Nature cell biology 2017, 19(7):820-832.

19. Schaffert N, Hossbach M, Heintzmann R, Achsel T, Lührmann R: RNAi knockdown of hPrp31 leads to an accumulation of U4/U6 di-snRNPs in Cajal bodies. The EMBO journal 2004, 23(15):3000-3009.

20. Makarov EM, Makarova OV, Achsel T, Lührmann R: The human homologue of the yeast splicing factor prp6p contains multiple TPR elements and is stably associated with the U5 snRNP via proteinprotein interactions. Journal of molecular biology 2000, 298(4):567-575.

21. Adler AS, McCleland ML, Yee S, Yaylaoglu M, Hussain S, Cosino E, Quinones G, Modrusan Z, Seshagiri S, Torres E et al: An integrative analysis of colon cancer identifies an essential function for PRPF6 in tumor growth. Genes \& development 2014, 28(10):1068-1084.

22. Wheway G, Nazlamova L, Meshad N, Hunt S, Jackson N, Churchill A: A Combined in silico, in vitro and Clinical Approach to Characterize Novel Pathogenic Missense Variants in PRPF31 in Retinitis Pigmentosa. Front Genet 2019, 10:248.

23. Liu W, Wang C, Wang S, Zeng K, Wei S, Sun N, Sun G, Wang M, Zou R, Liu W et al: PRPF6 promotes androgen receptor/androgen receptor-variant 7 actions in castration-resistant prostate cancer cells. International journal of biological sciences 2021, 17(1):188-203.

24. Song H, Sun N, Lin L, Wei S, Zeng K, Liu W, Wang C, Zhong X, Wang M, Wang S et al: Splicing factor PRPF6 upregulates oncogenic androgen receptor signaling pathway in hepatocellular carcinoma. Cancer science 2020, 111(10):3665-3678.

25. Wahl MC, Will CL, Lührmann R: The spliceosome: design principles of a dynamic RNP machine. Cell 2009, 136(4):701-718.

26. Tanackovic G, Ransijn A, Ayuso C, Harper S, Berson EL, Rivolta C: A missense mutation in PRPF6 causes impairment of pre-mRNA splicing and autosomal-dominant retinitis pigmentosa. American journal of human genetics 2011, 88(5):643-649.

27. Ren J, Chen Y, Song H, Chen L, Wang R: Inhibition of ZEB1 reverses EMT and chemoresistance in docetaxel-resistant human lung adenocarcinoma cell line. J Cell Biochem 2013, 114(6):1395-1403.

28. Ashrafizadeh M, Mirzaei S, Hashemi F, Zarrabi A, Zabolian A, Saleki H, Sharifzadeh SO, Soleymani L, Daneshi S, Hushmandi $\mathrm{K}$ et al: New insight towards development of paclitaxel and docetaxel resistance in cancer cells: EMT as a novel molecular mechanism and therapeutic possibilities. Biomedicine \& pharmacotherapy = Biomedecine \& pharmacotherapie 2021, 141:111824.

29. Mozzetti S, Ferlini C, Concolino P, Filippetti F, Raspaglio G, Prislei S, Gallo D, Martinelli E, Ranelletti FO, Ferrandina $\mathrm{G}$ et al: Class III beta-tubulin overexpression is a prominent mechanism of paclitaxel resistance in ovarian cancer patients. Clinical cancer research: an official journal of the American Association for Cancer Research 2005, 11(1):298-305.

30. Pang W, Zhai M, Wang Y, Li Z: Long noncoding RNA SNHG16 silencing inhibits the aggressiveness of gastric cancer via upregulation of microRNA-628-3p and consequent decrease of NRP1. Cancer management and research 2019, 11:7263-7277. 
31. Zhou C, Zhao J, Liu J, Wei S, Xia Y, Xia W, Bi Y, Yan Z, Huang H: LncRNA SNHG16 promotes epithelialmesenchymal transition via down-regulation of DKK3 in gastric cancer. Cancer biomarkers: section $A$ of Disease markers 2019, 26(4):393-401.

32. Chen ZY, Wang XY, Yang YM, Wu MH, Yang L, Jiang DT, Cai H, Peng Y: LncRNA SNHG16 promotes colorectal cancer cell proliferation, migration, and epithelial-mesenchymal transition through miR124-3p/MCP-1. Gene therapy 2020.

33. Hu YL, Feng Y, Chen YY, Liu JZ, Su Y, Li P, Huang H, Mao QS, Xue WJ: SNHG16/miR-6053p/TRAF6/NF-kB feedback loop regulates hepatocellular carcinoma metastasis. Journal of cellular and molecular medicine 2020, 24(13):7637-7651.

34. Shao M, Yu Z, Zou J: LncRNA-SNHG16 Silencing Inhibits Prostate Carcinoma Cell Growth, Downregulate GLUT1 Expression and Reduce Glucose Uptake. Cancer management and research 2020, 12:1751-1757.

35. Wu W, Guo L, Liang Z, Liu Y, Yao Z: Lnc-SNHG16/miR-128 axis modulates malignant phenotype through WNT/ß-catenin pathway in cervical cancer cells. Journal of Cancer 2020, 11(8):2201-2212.

36. Tao L, Wang X, Zhou Q: Long noncoding RNA SNHG16 promotes the tumorigenicity of cervical cancer cells by recruiting transcriptional factor SPI 1 to upregulate PARP9. Cell biology international 2020, 44(3):773-784.

37. Li W, Tanikawa T, Kryczek I, Xia H, Li G, Wu K, Wei S, Zhao L, Vatan L, Wen B et al: Aerobic Glycolysis Controls Myeloid-Derived Suppressor Cells and Tumor Immunity via a Specific CEBPB Isoform in Triple-Negative Breast Cancer. Cell metabolism 2018, 28(1):87-103.e106.

38. Wang F, Gao Y, Tang L, Ning K, Geng N, Zhang H, Li Y, Li Y, Liu F, Li F: A novel PAK4-CEBPB-CLDN4 axis involving in breast cancer cell migration and invasion. Biochemical and biophysical research communications 2019, 511(2):404-408.

39. Liu D, Zhang XX, Li MC, Cao CH, Wan DY, Xi BX, Tan JH, Wang J, Yang ZY, Feng XX et al: C/EBPB enhances platinum resistance of ovarian cancer cells by reprogramming H3K79 methylation. Nature communications 2018, 9(1):1739.

40. Guo LL, Wang SF: Downregulated Long Noncoding RNA GAS5 Fails to Function as Decoy of CEBPB, Resulting in Increased GDF15 Expression and Rapid Ovarian Cancer Cell Proliferation. Cancer biotherapy \& radiopharmaceuticals 2019, 34(8):537-546.

41. El-Arabey AA, Abdalla M, Abd-Allah AR: GATA3 and stemness of high-grade serous ovarian carcinoma: novel hope for the deadliest type of ovarian cancer. Human cell 2020, 33(3):904-906.

42. Chen HJ, Huang RL, Liew PL, Su PH, Chen LY, Weng YC, Chang CC, Wang YC, Chan MW, Lai HC: GATA3 as a master regulator and therapeutic target in ovarian high-grade serous carcinoma stem cells. International journal of cancer 2018, 143(12):3106-3119.

43. El-Arabey AA, Denizli M, Kanlikilicer P, Bayraktar R, Ivan C, Rashed M, Kabil N, Ozpolat B, Calin GA, Salama SA et al: GATA3 as a master regulator for interactions of tumor-associated macrophages with high-grade serous ovarian carcinoma. Cellular signalling 2020, 68:109539. 
44. Li B, Jin X, Meng H, Hu B, Zhang T, Yu J, Chen S, Guo X, Wang W, Jiang W et al: Morin promotes prostate cancer cells chemosensitivity to paclitaxel through miR-155/GATA3 axis. Oncotarget 2017, 8(29):47849-47860.

45. Li W, Zhang Z, Liu X, Cheng X, Zhang Y, Han X, Zhang Y, Liu S, Yang J, Xu B et al: The FOXN3-NEAT1SIN3A repressor complex promotes progression of hormonally responsive breast cancer. The Journal of clinical investigation 2017, 127(9):3421-3440.

46. Ule J, Blencowe BJ: Alternative Splicing Regulatory Networks: Functions, Mechanisms, and Evolution. Mol Cel/ 2019, 76(2):329-345.

\section{Tables}

Due to technical limitations, table 1,2 is only available as a download in the Supplemental Files section.

Figures

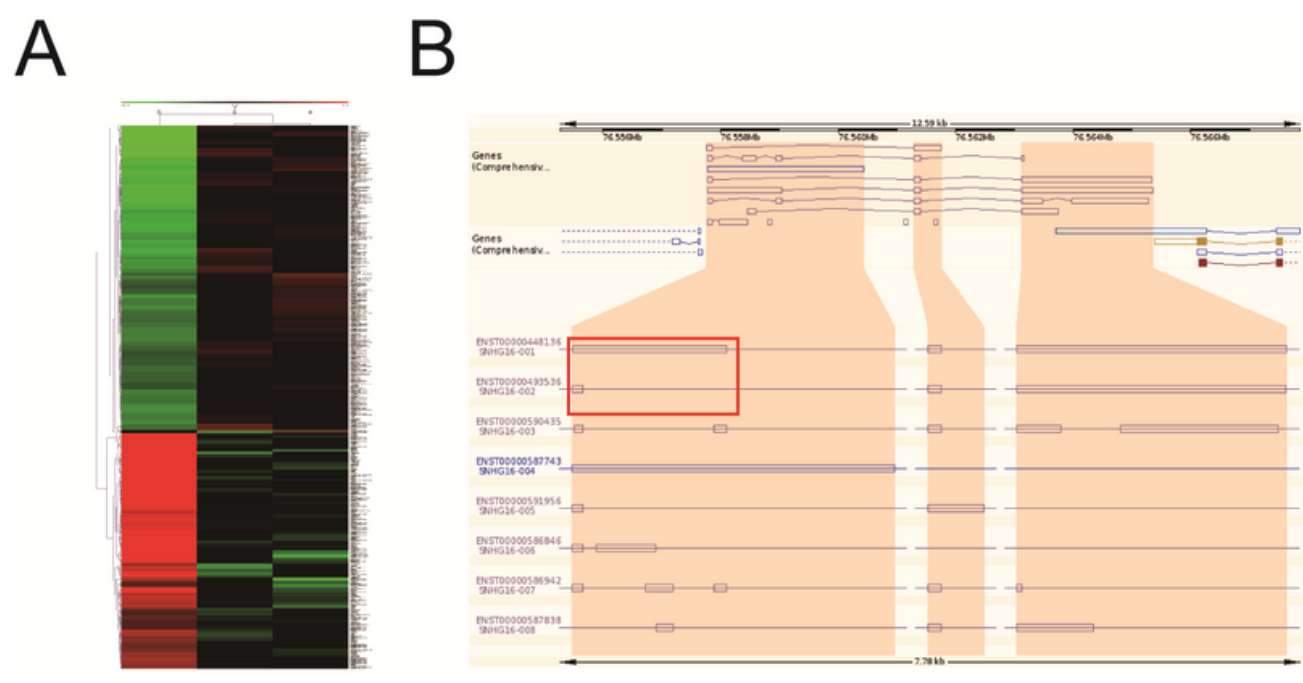

D

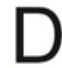

- Chemosensitivity

- Chemoresistance

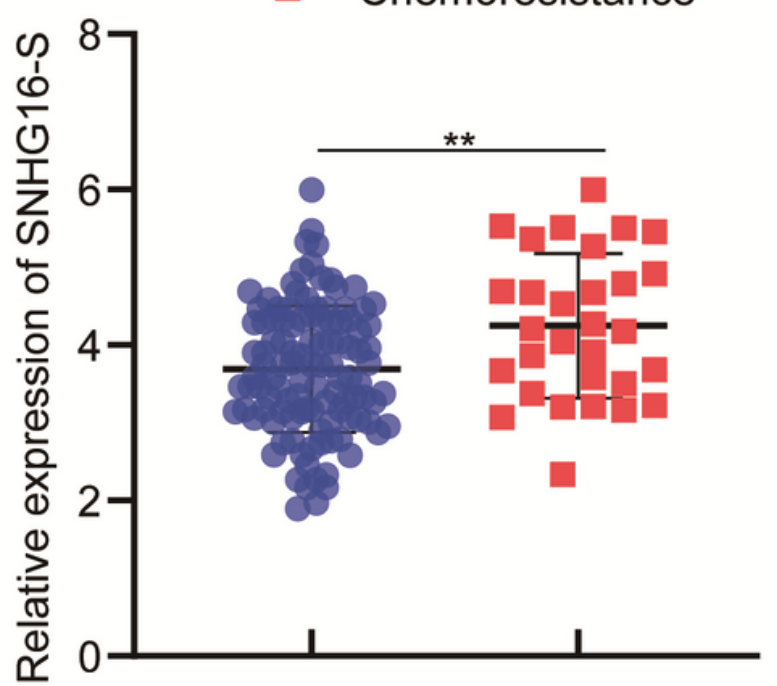

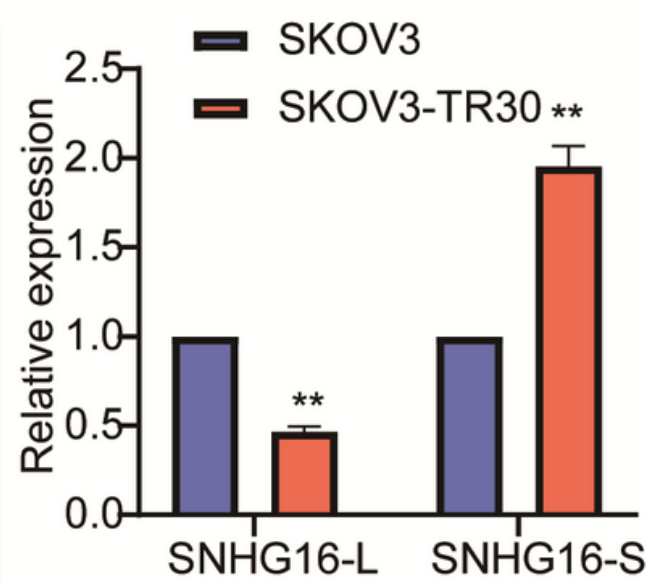

- Chemosensitivity

- Chemoresistance

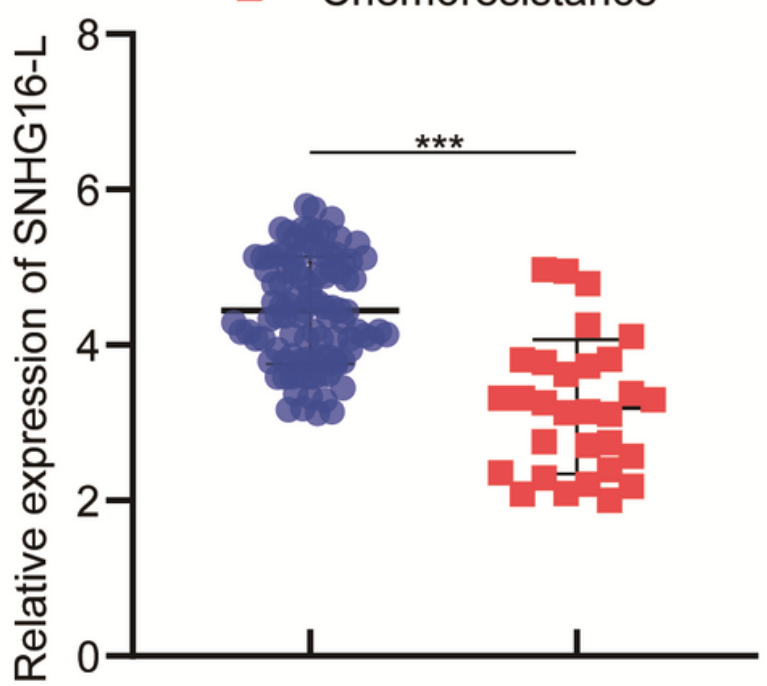


Figure 1

SNHG16-L/S were expressed opposite in OC A. LncRNA arrays analysis in SKOV3 and SKOV3-TR30. B. ENSEMBL's annotation showed the difference of SNHG16-L/S. C. SNHG16-L/S expression in SKOV3 and SKOV3-TR30 cells analyzed by RT-PCR. D. SNHG16-L/S expression in OC tissues, chemosensitivity group $(\mathrm{N}=102)$ and chemoresistance $(\mathrm{N}=30) .\left({ }^{\star} \mathrm{p}<0.05,{ }^{*} \mathrm{p}<0.01,{ }^{\star \star *} \mathrm{p}<0.001\right)$

A
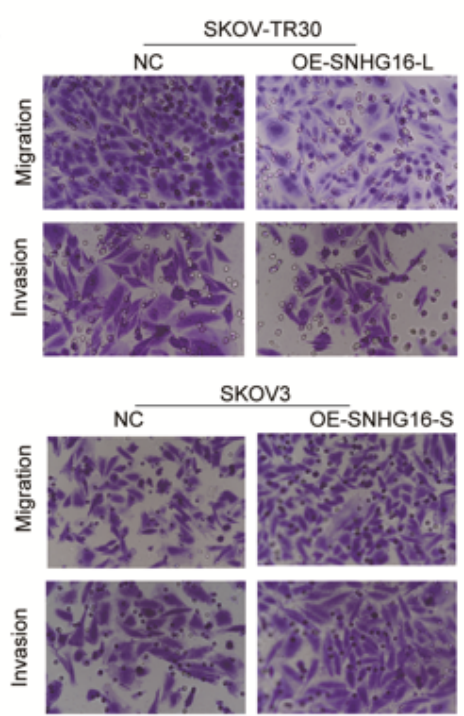

$\mathrm{C}$
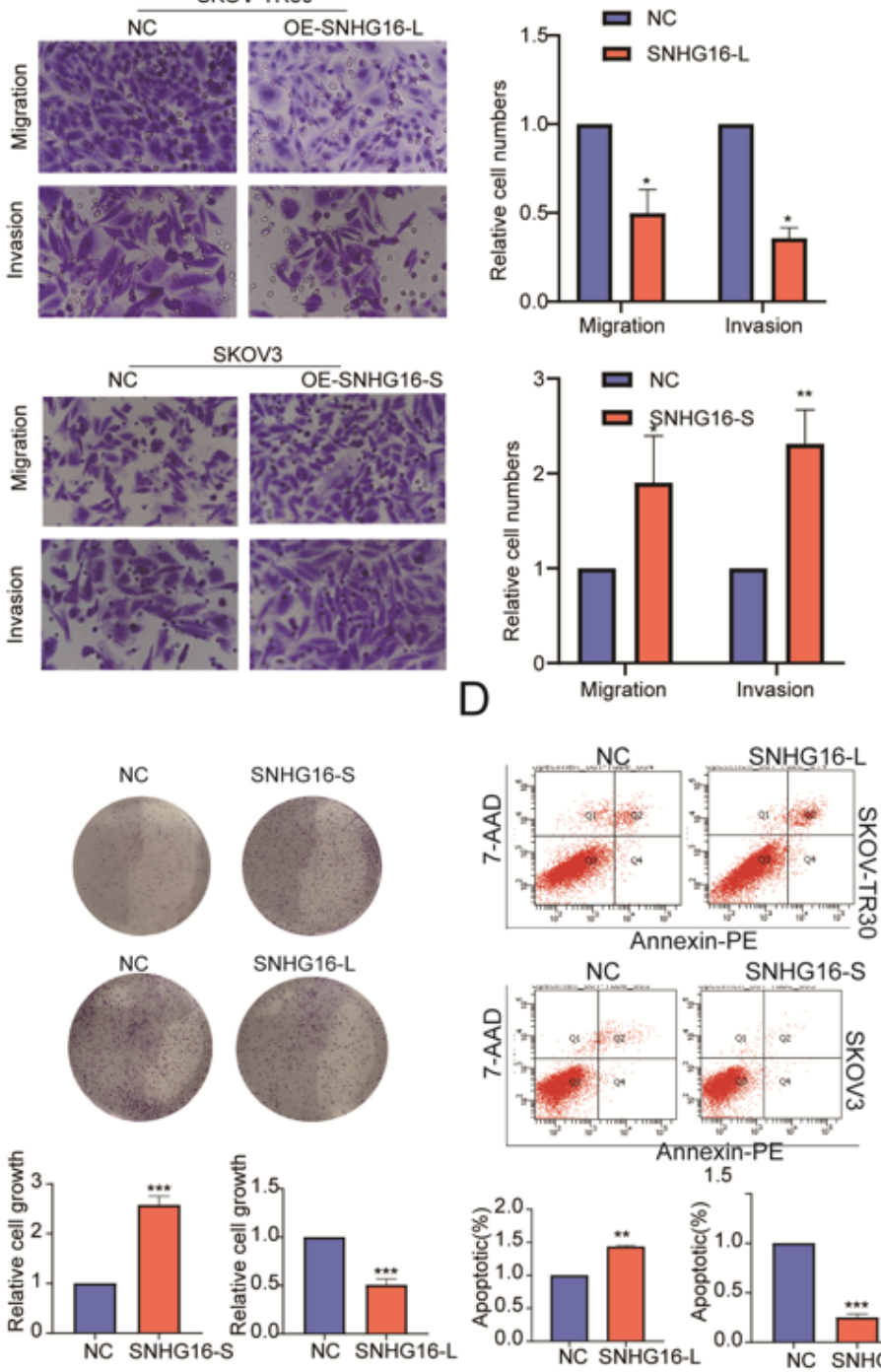

$\mathrm{F}$

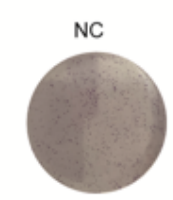

SNHG16-S

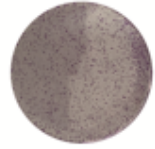

SNHG16-L
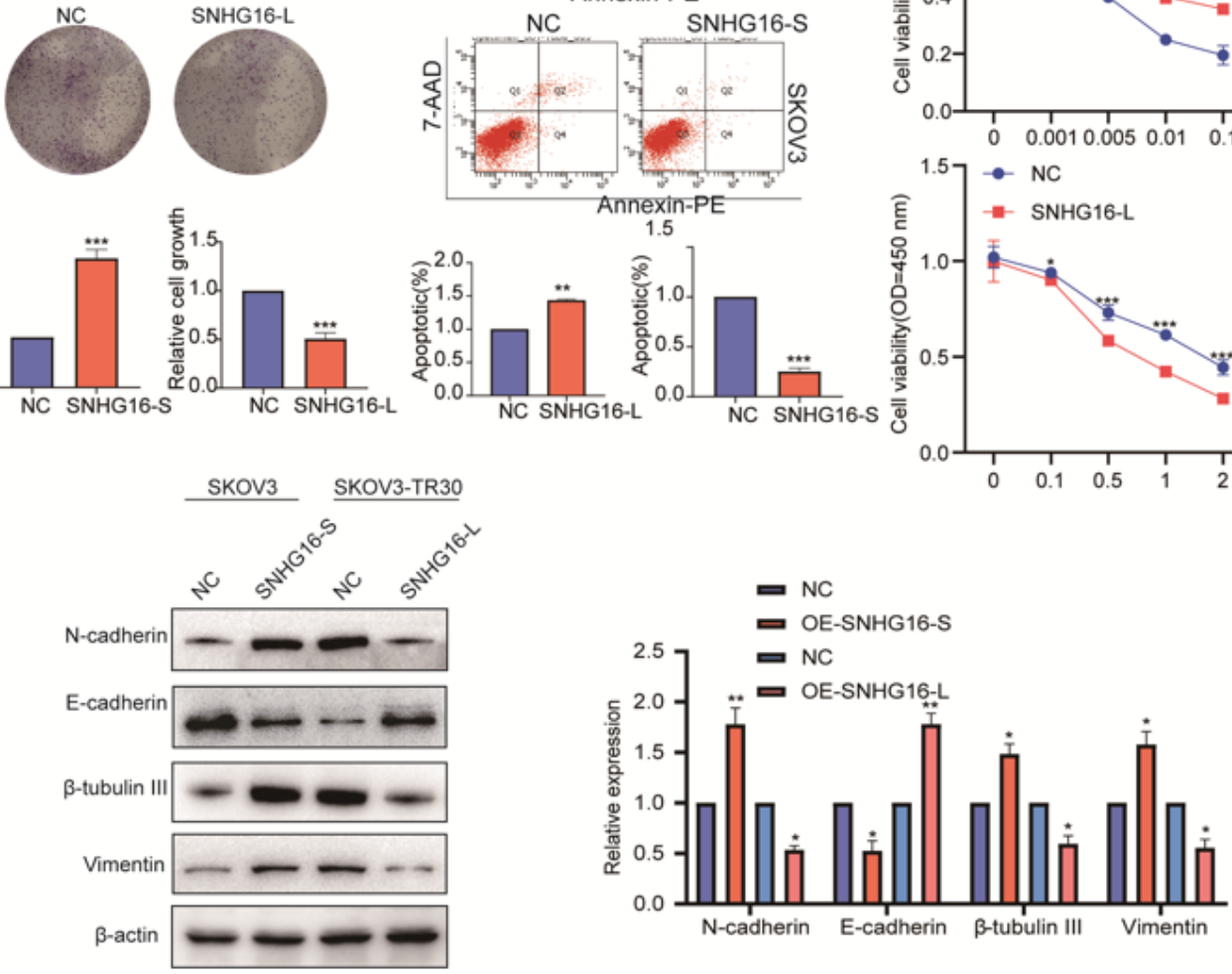

Figure 2

B

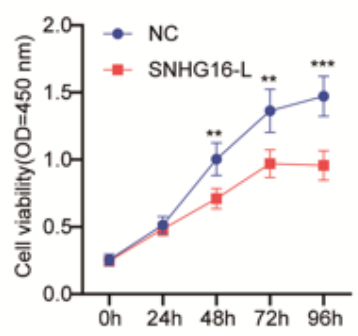

E
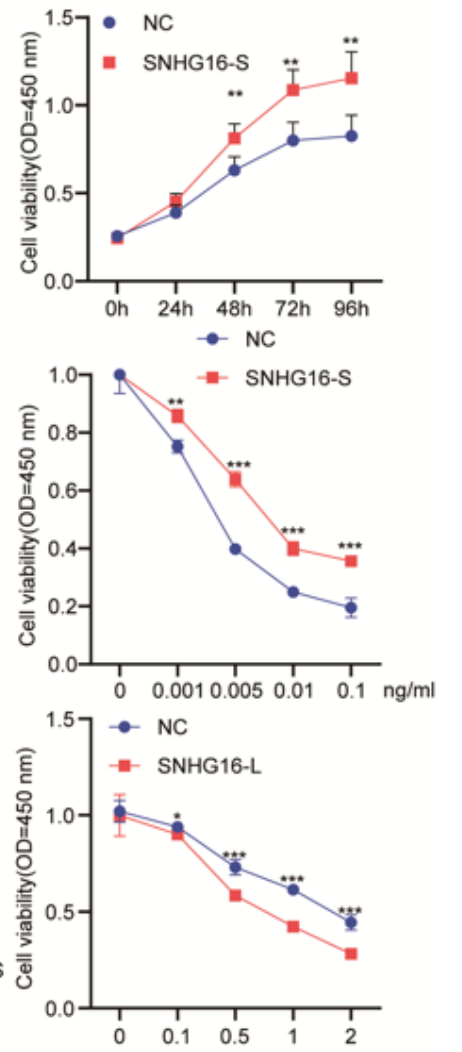
SNHG16-L/S had different effect on the tumorigenesis, and PTX-resistance of OC A. Overexpression of SNHG16-S in SKOV3 and overexpression of SNHG16-L in SKOV3-TR30, transwell assays were used to detect cell migration and invasion. B. Cell growth was determined by CCK8 assays. C. Colony formation assay was used to detect cell growth. D. The flow cytometry analysis was used to detect cell apoptosis. E. PTX-resistance were examined by CCK8 cell viability assays. F. N-cadherin, E-cadherin, $\beta$-tubulin III and Vimentin analyzed by western blot. ( $\left.\mathrm{k}<<0.05,{ }^{*} \mathrm{p}<0.01,{ }^{* \star *} \mathrm{p}<0.001\right)$.

A

OV ENSG00000163597

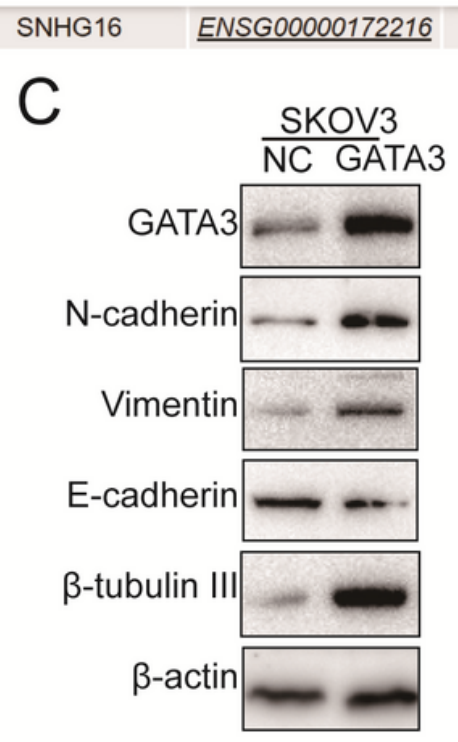

CEBPB ENSG00000107485

GATA3

$+$

B
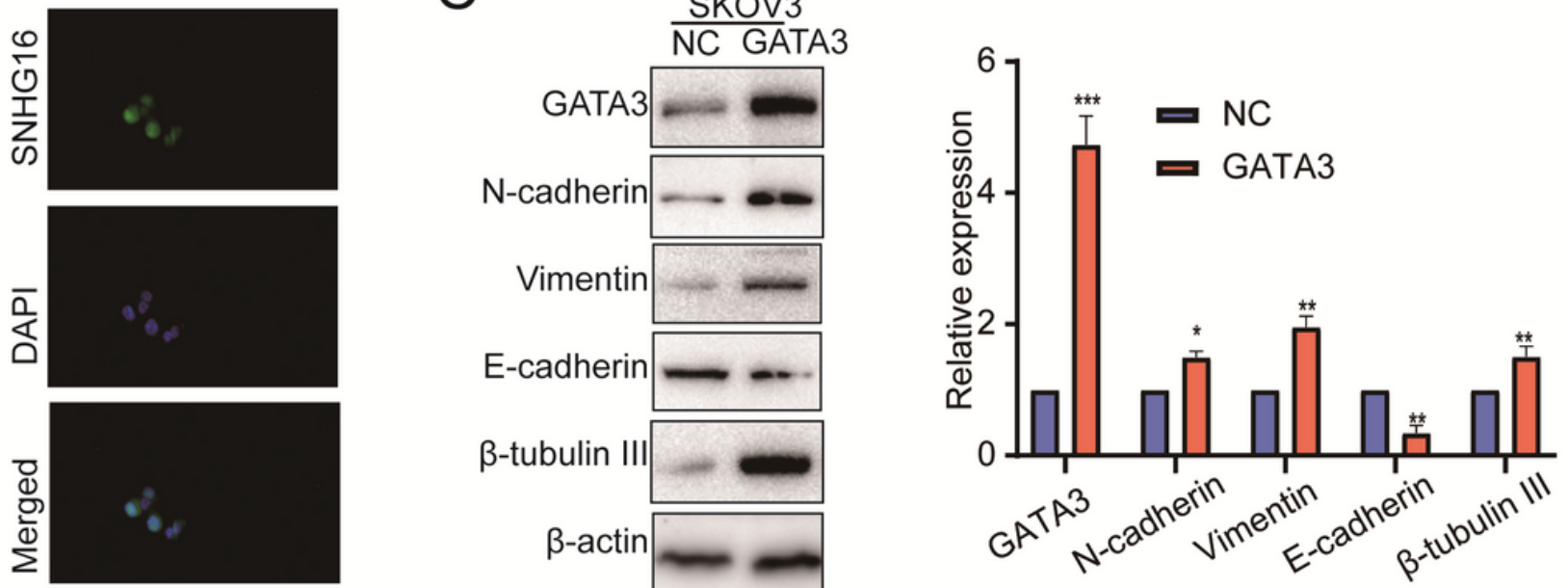

$\mathrm{D}$

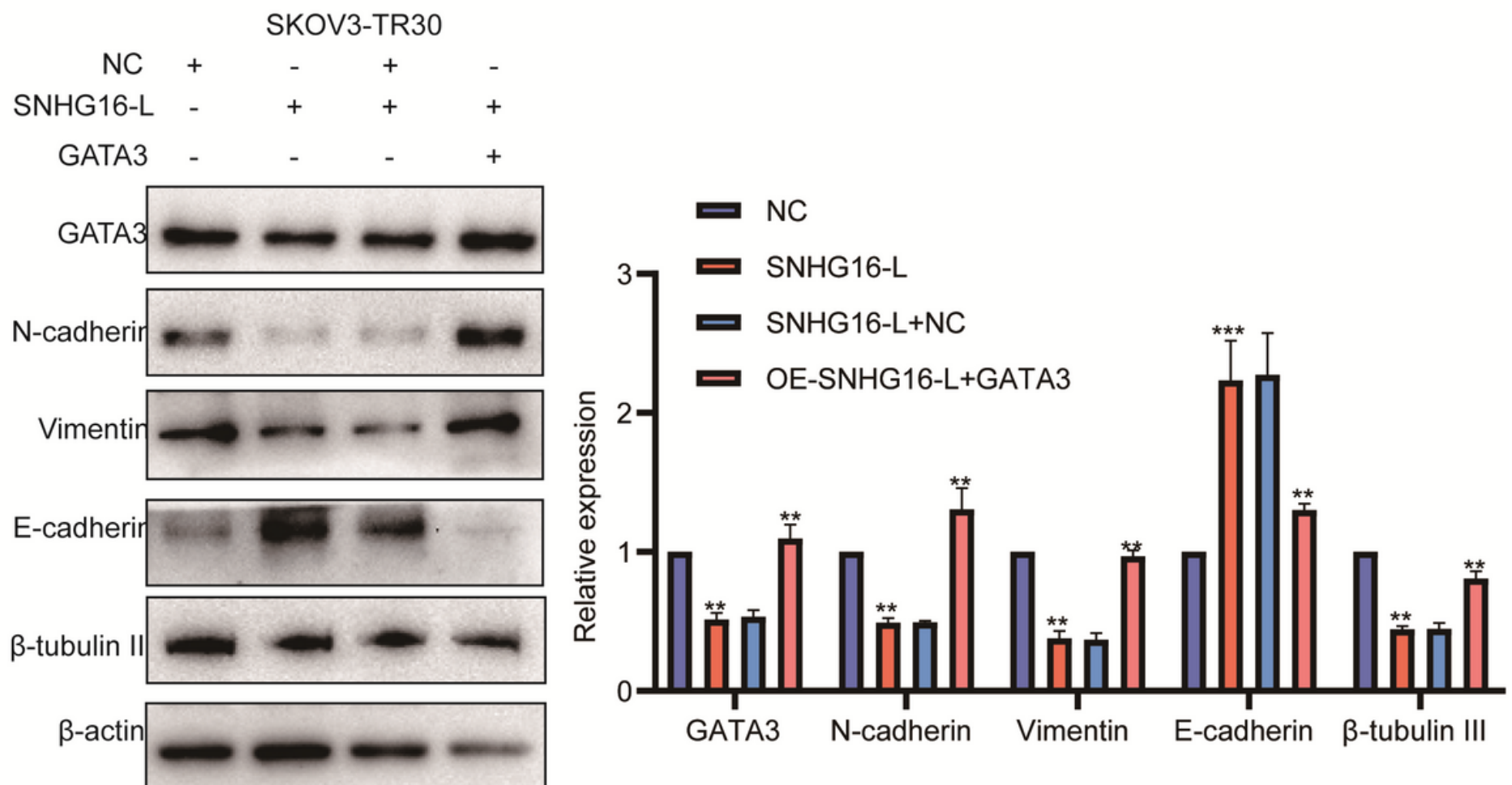

\section{Figure 3}

SNHG16-L downregulated GATA3 expression to inhibit EMT and PTX-resistance of OC A. The possible function mechanism of SNHG16 in OC predicted on LncMAP database. B. SNHG16 location was detected 
by FISH. C. GATA3, N-cadherin, vimentin, E-cadherin and $\beta$-tubulin III were detected by western blot after overexpression of GATA3. D. Those proteins were examined by western blot after overexpression of SNHG16-L and further overexpression of GATA3 in SKOV3-TR30. ( $\left.{ }^{*} p<0.05,{ }^{*} p<<0.01,{ }^{* *} \mathrm{p}<0.001\right)$

A

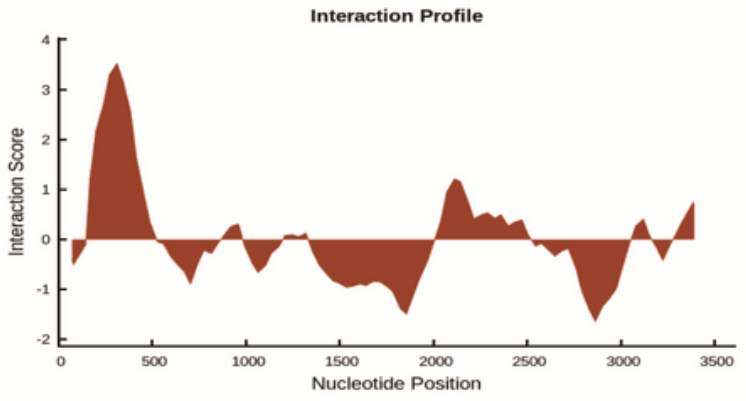

Interaction Matrix
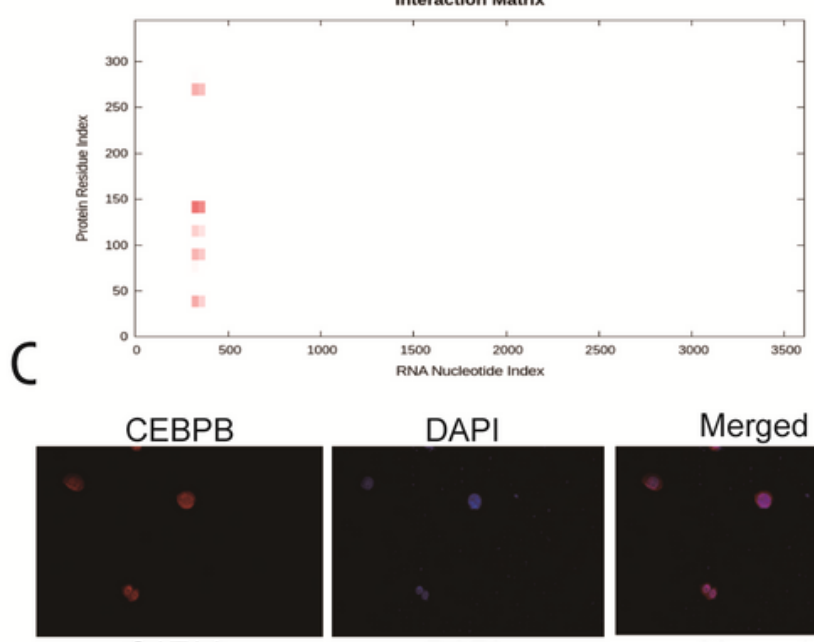

GATA3

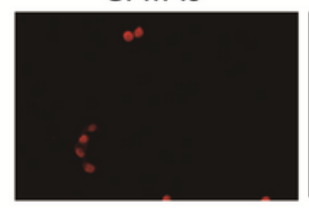

$E$

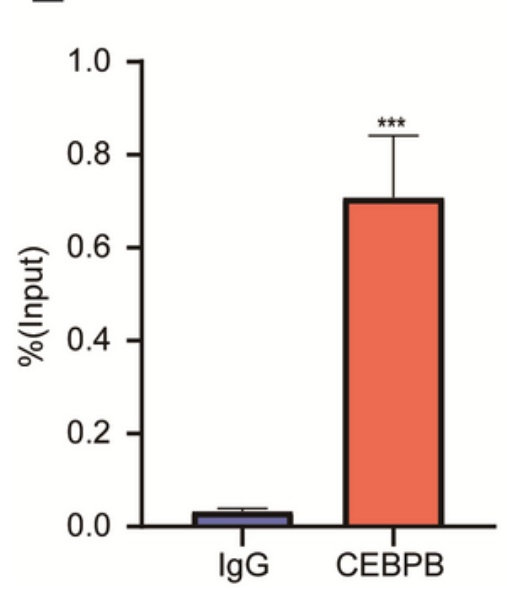

DAPI

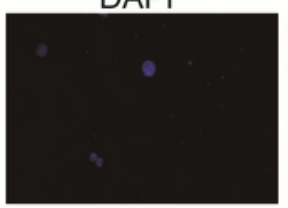

DAPI

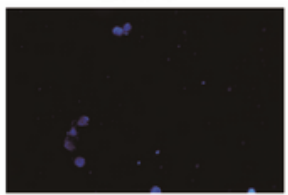

$\mathrm{F}$
$\mathrm{B}$

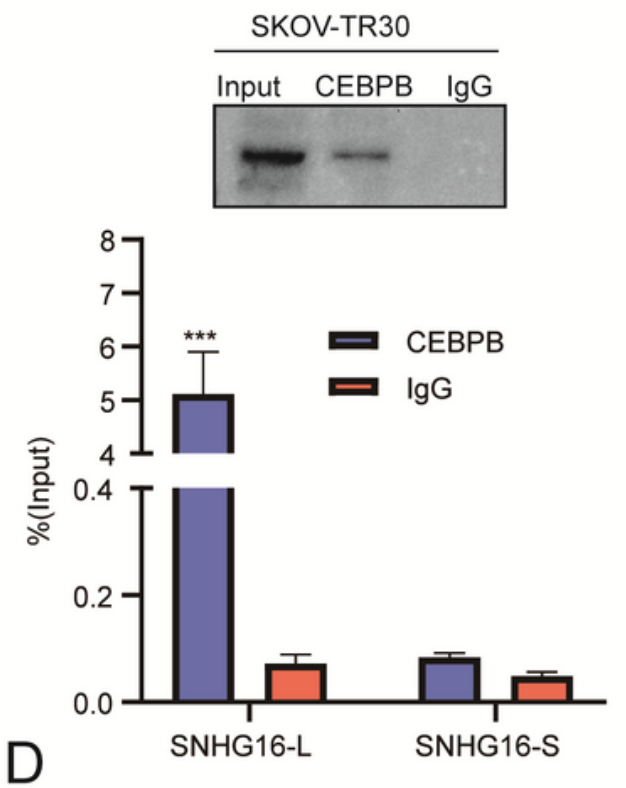

\section{Merged}

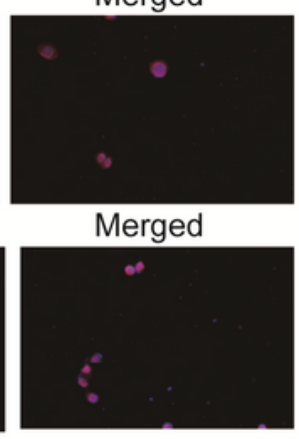

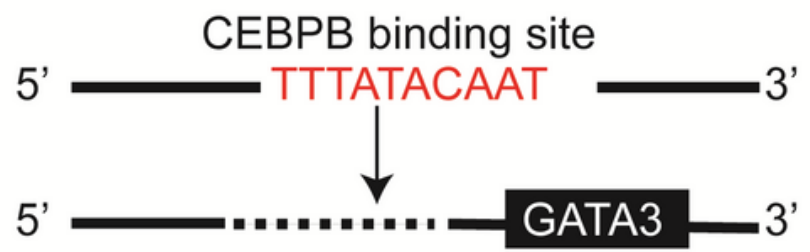

G
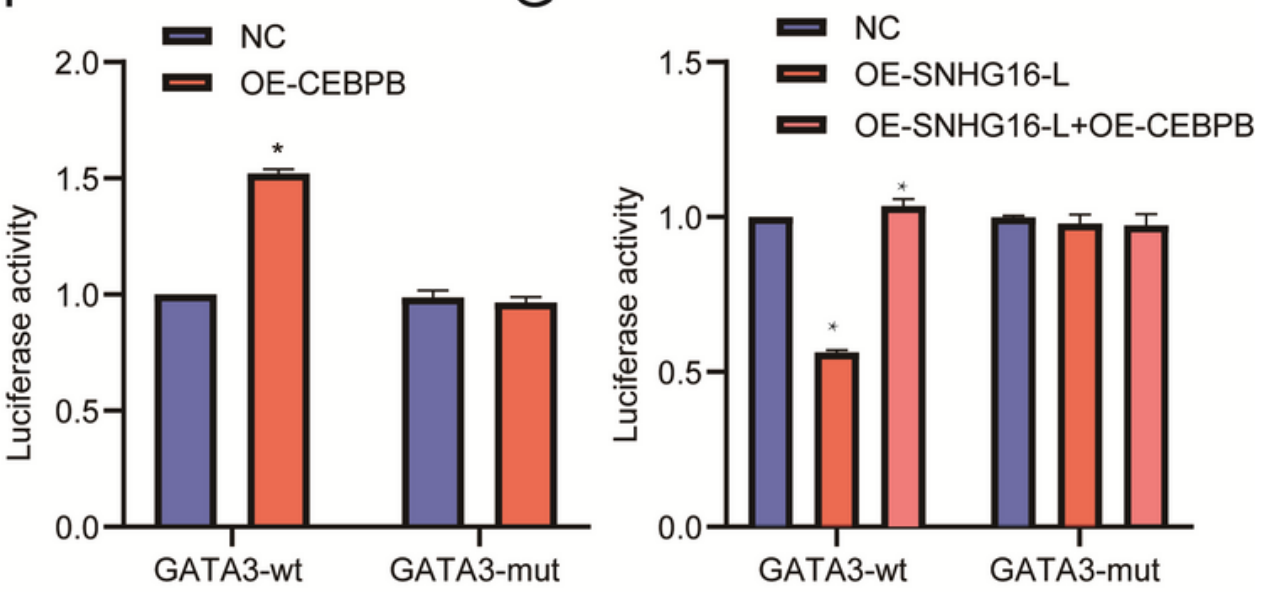

Figure 4

SNHG16-L combined with CEBPB and inhibited GATA3's transcriptional activity in OC A. The analysis of RNA interaction predicted on CatRAPID omics the putative binding sequence of SNHG16-L to CEBPB 
protein. B. RIP was performed by using the CEBPB antibody in SKOV3-TR30 cells and the levels of coprecipitated RNAs were determined by RT-PCR. C. Immunofluorescence was used to detect the expression of CEBPB and GATA3. D. A schematic illustration of the proximal region between CEBPB and GATA3 promoter. E. ChIP detection in SKOV3-TR30 using antibody of CEBPB were carried out to verify binding between CEBPB and GATA3 promoter and the levels were analyzed by RT-PCR. F. Dual-luciferase reporter gene assay were performed in 293T cells which were transfected with GATA3-WT and GATA3-MUT. Those cells further transfected with OE-CEBPB. G. Dual-luciferase reporter gene assay was performed after those cells were further transfected with OE-SNHG16-L. $\left({ }^{*} p<0.05,{ }^{* *} p<0.01,{ }^{* *} p<0.001\right)$

\section{A}

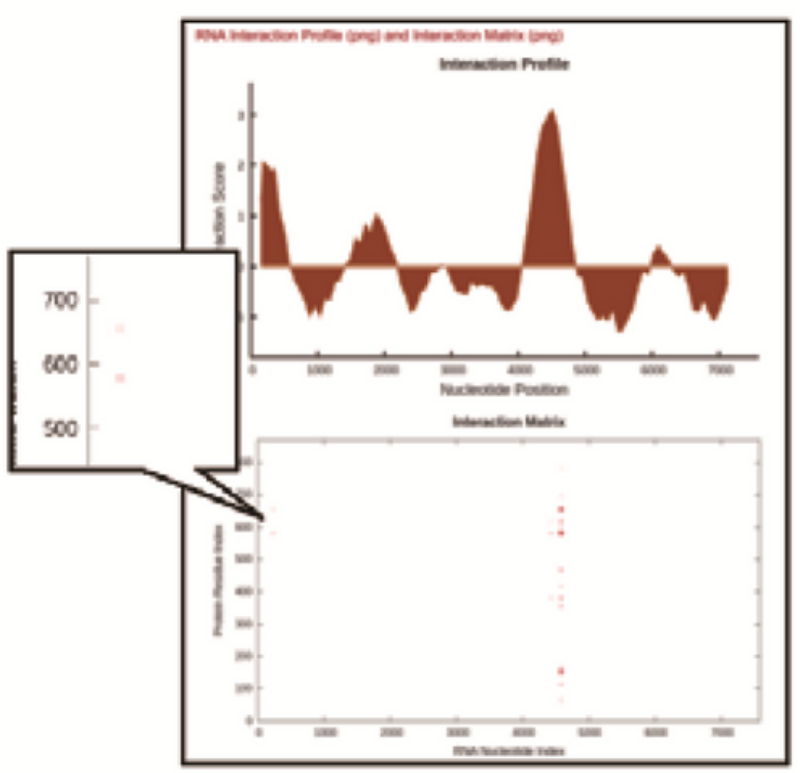

C

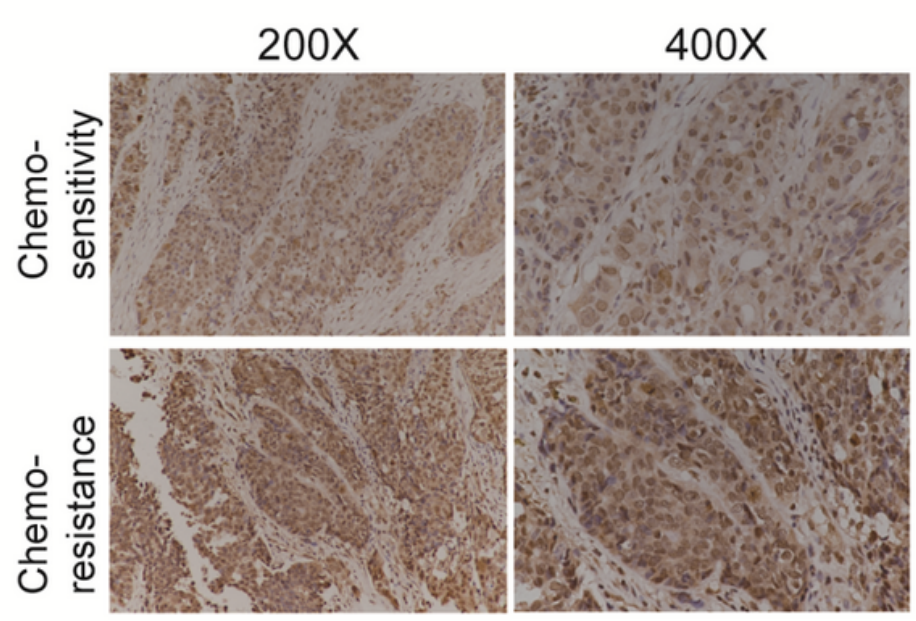

B
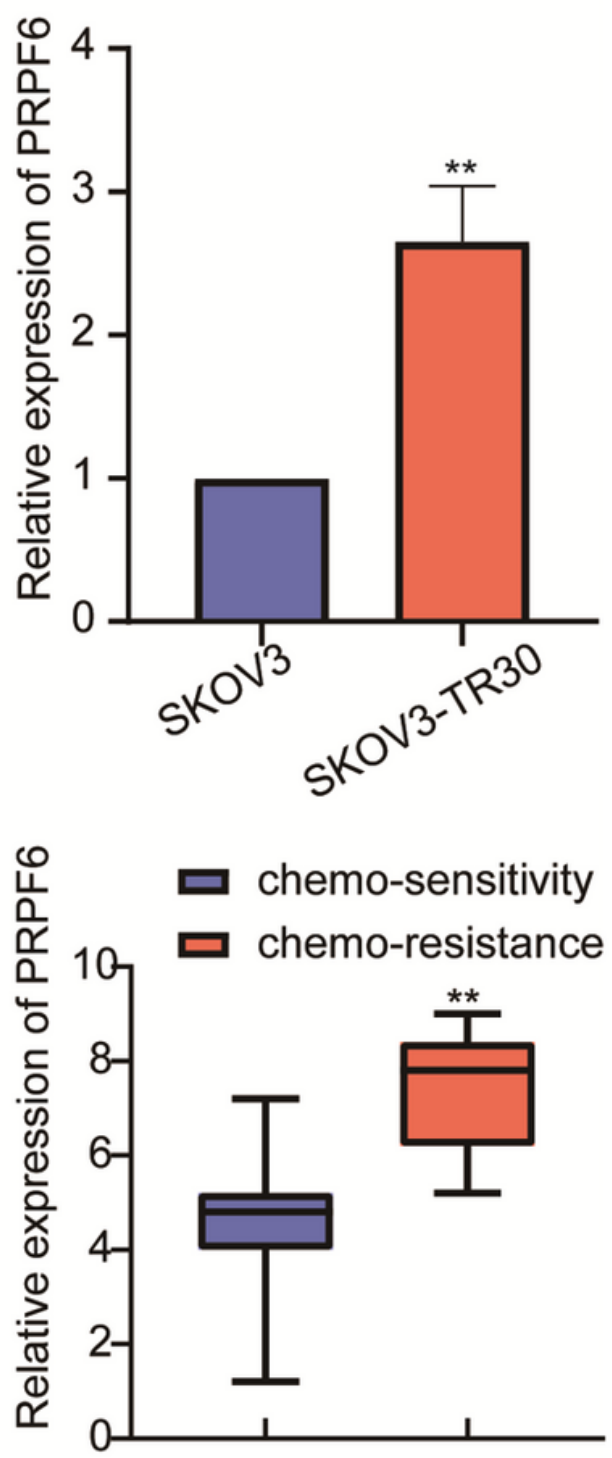

\section{Figure 5}

PRPF6 was upregulated in chemo-resistant tissues and was related to FIGO stage, differentiation and lymph node metastasis of OC A. The analysis of RNA interaction predicted on CatRAPID omics the putative binding sequence of SNHG16 pre-mRNA to PRPF6 protein. B. PRPF6 expression in SKOV3 and 
SKOV3-TR30 cells were analyzed by RT-PCR. C. PRPF6 expression in OC tissues were examined by IHC. $\left({ }^{*} p<0.05,{ }^{* \star} p<0.01, * \star \star p p<0.001\right)$

A

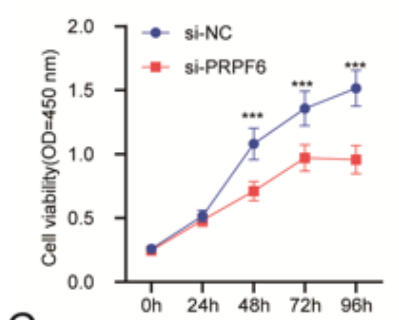

C
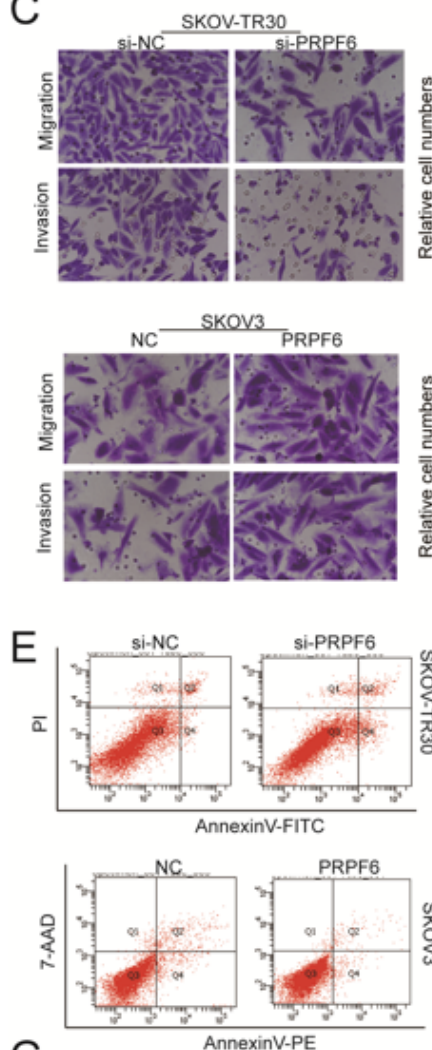

G

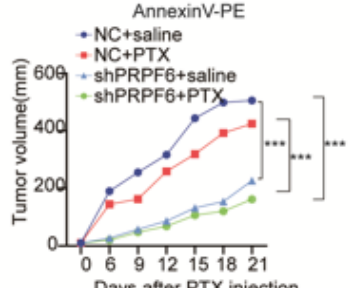

$\mathrm{H}$

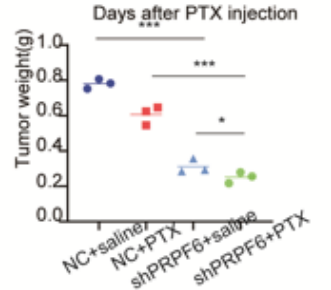

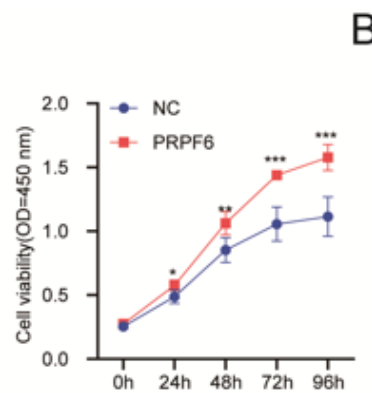

B
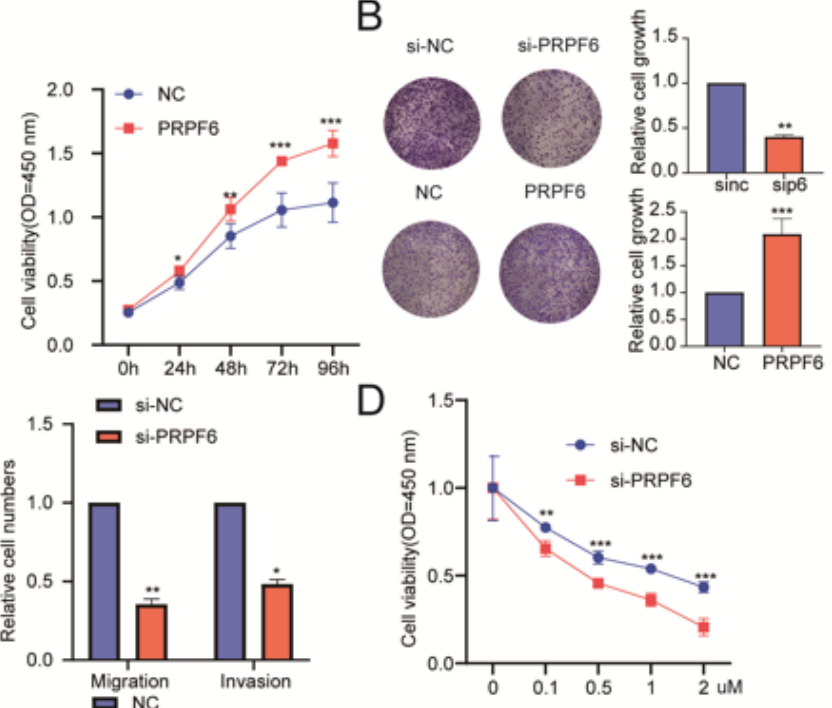

D
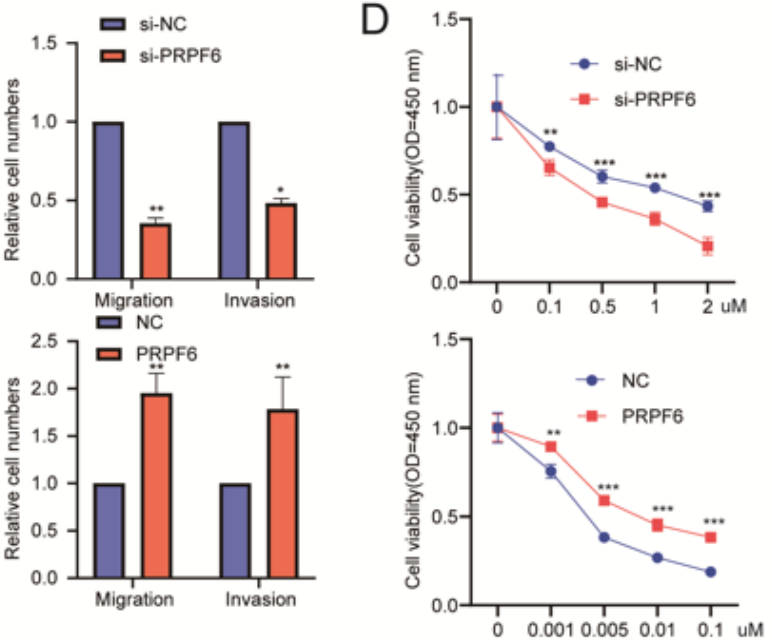

$\mathrm{F}$
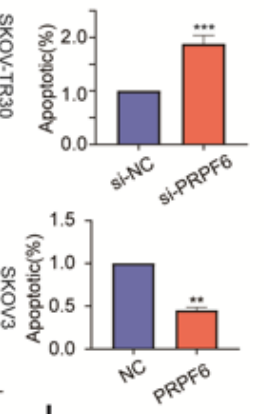

shPRPF6+saline

shPRPF6+PTX

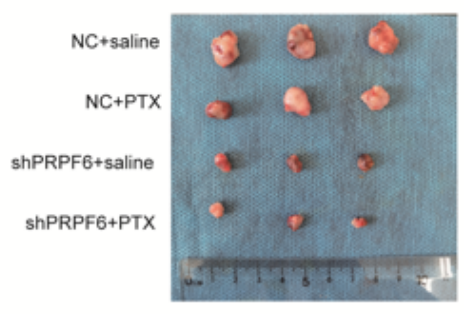

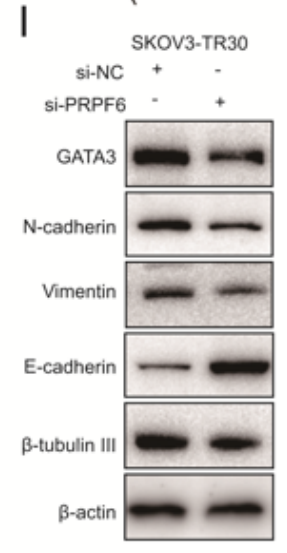

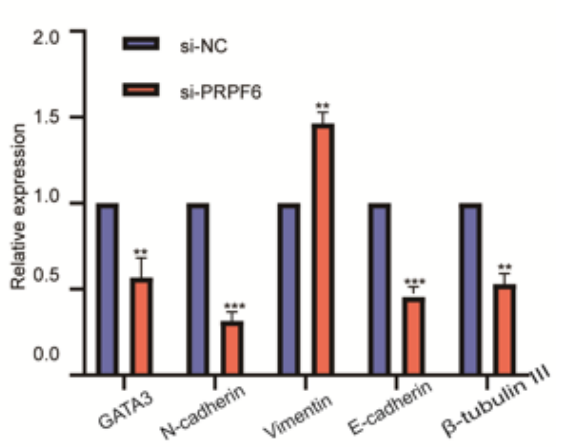

Figure 6

PRPF6 promoted tumorigenesis, EMT and PTX-resistance in OC cells and in vivo A. Silencing PRPF6 in SKOV3-TR30 and overexpression of PRPF6 in SKOV3 cells. Cell growth was determined by CCK8 assays. B. Colony formation assays were used to detect cell growth. C. Transwell assays were used to detect cell 
migration and invasion. D. PTX-resistance were examined by CCK8 cell viability assays. E. The flow cytometry analysis was used to detect cell apoptosis. F. SKOV3 cells were stably transfected with lentivirus containing siPRPF6 sequence. The cells were injected in 4-weeks-old female BALB/cA-nu Mice( $\mathrm{N}=3 /$ group). PTX $(20 \mathrm{mg} / \mathrm{kg}$ ) or saline was injected into tumor every 3 days for 3 weeks. Images of tumors were pictured. G. Tumor volume was measured every 3 days (tumor volume=length $\times$ width $2 / 2$ ). $\mathrm{H}$. Tumor weight was measured after sacrifice. G. IHC was used to detect PRPF6 expression in tumor tissues. I. GATA3, N-cadherin, E-cadherin, $\beta$-tubulin III and vimentin were detected by western blot. ${ }^{\star} \mathrm{p}<$ $0.05, * \star p<0.01, * \star \star p<0.001)$ 
B
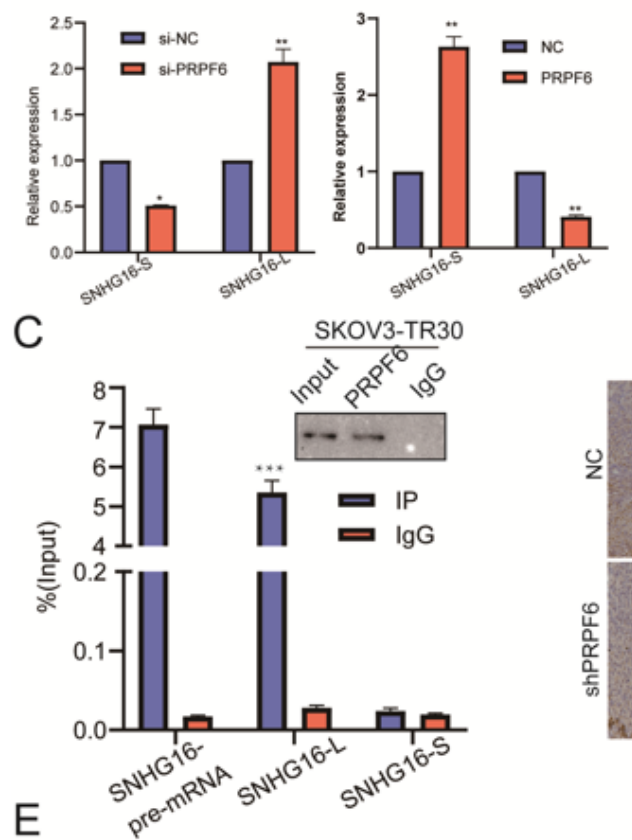

$D_{200 x}$
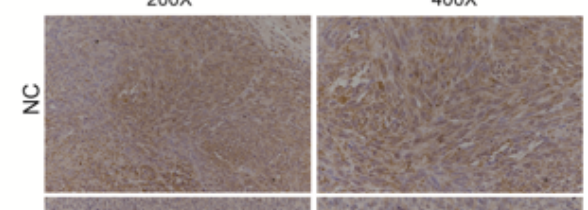

$\frac{6}{4}$
$\frac{0}{\alpha}$
$\frac{\alpha}{0}$
$\frac{c}{5}$
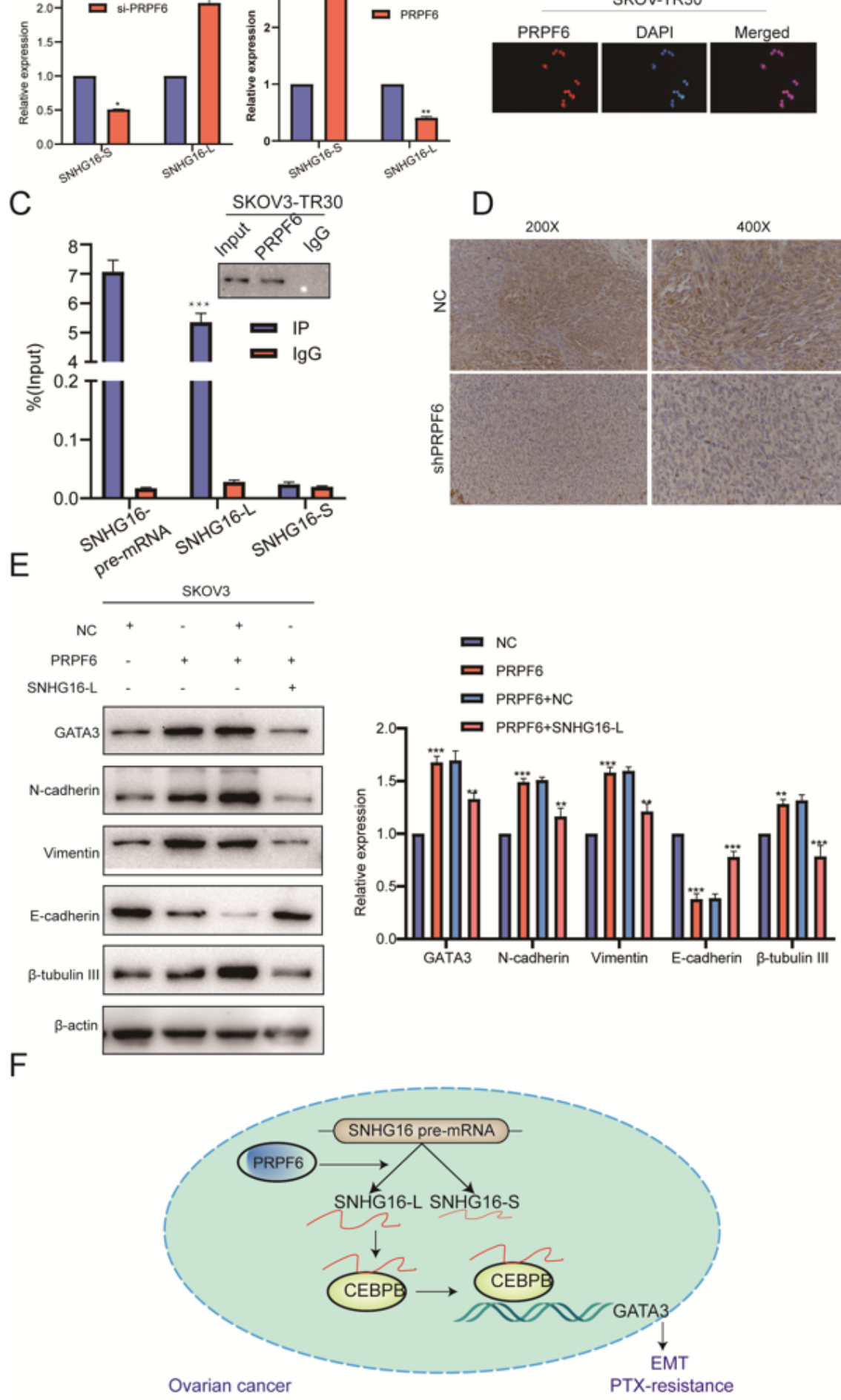

Figure 7

PRPF6 induced AS of SNHG16 to upregulate GATA3 expression A. siPRPF6 were transfected in SKOV3TR30 and OE-PRPF6 in SKOV3, and RT-PCT were used to detect SNHG16-L/S expression. B. Immunofluorescence detected the expression of PRPF6. C. RIP test using the PRPF6-specific antibody was conducted in over expressed PRPF6 SKOV3-TR30 cells and the co-precipitated RNAs were determined by RT-PCR. D. IHC was used to detect GATA3 expression in mice tumor tissues. E. GATA3, N- 
cadherin, E-cadherin, $\beta$-tubulin III and vimentin expression was examined by western blot after overexpression of PRPF6 and SNHG16-L. F. A scheme of the proposed mechanisms, PRPF6 induced AS of SNHG16 to promote EMT and PTX-resistance via SNHG16-L/CEBPB/GATA3 axis in OC. $\left({ }^{*} p<0.05\right.$, **p $<0.01, * \star \star p<0.001)$

\section{Supplementary Files}

This is a list of supplementary files associated with this preprint. Click to download.

- Table1.xlsx

- Table2.xlsx

- Supplementaryfig.1.tif 